\title{
Global dynamics of an nonautonomous SEIRS epidemic model with vaccination and nonlinear incidence
}

\author{
Long Zhang ${ }^{1}$, Xiaolin Fan ${ }^{2}$, and Zhidong Teng ${ }^{1}$ \\ ${ }^{1}$ Xinjiang University \\ ${ }^{2}$ Xinjiang Institute of Engineering
}

May 5, 2020

\begin{abstract}
In this paper, a class of nonautonomous SEIRS epidemic models with vaccination and nonlinear incidence is investigated. Under some quite weak assumptions, a couple of new threshold values in the form of integral, i.e., $\$ R_{-} \_1 \$, \$ R^{\wedge *} \_1 \$, \$ R_{-} 2 \$$ and $\$ R^{\wedge *} \_2 \$$ on the extinction and permanence of disease for the model are established. As special cases of our model, the autonomous, periodic and almost periodic circumstances are discussed respectively. The nearly necessary and sufficient criteria of threshold on the extinction and permanence of disease for above cases are obtained as well. Numerical examples and simulations are presented to illustrate the analytic results.
\end{abstract}

\section{Hosted file}

MMA-ZFT.pdf available at https://authorea.com/users/301064/articles/430843-global-dynamicsof-an-nonautonomous-seirs-epidemic-model-with-vaccination-and-nonlinear-incidence 
figures/seir3/seir3-eps-converted-to.pdf 
figures/seir4/seir4-eps-converted-to.pdf 
figures/seirsanwei3/seirsanwei3-eps-converted-to.pdf 
figures/seirsanwei4/seirsanwei4-eps-converted-to.pdf 
figures/seirsanwei31/seirsanwei31-eps-converted-to.pdf 
figures/seirsanwei32/seirsanwei32-eps-converted-to.pdf 
figures/seirsanwei41/seirsanwei41-eps-converted-to.pdf 
figures/seirsanwei42/seirsanwei42-eps-converted-to.pdf 
figures/u11/u11-eps-converted-to.pdf 
figures/u22/u22-eps-converted-to.pdf 
figures/z11/z11-eps-converted-to.pdf 
figures/z22/z22-eps-converted-to.pdf 